Special Issue: Characterization of Crack Tip Stress Field

\title{
Initiation and growth behavior of very-long microstructurally short fatigue cracks
}

\author{
P. Lorenzino, A. Navarro \\ Escuela Superior de Ingenieros, Universidad de Sevilla. Camino de los Descubrimientos s/n, 41092, Seville, Spain.
}

\begin{abstract}
The present paper describes a novel experimental technique recently presented that allows one to study interactions between the crack and microstructural barriers with an unprecedented level of ease and detail. The method consists in increasing the grain size of Al1050 Aluminium alloy until the centimetre scale by applying a series of mechanical and heat treatments. Once the thermo-mechanical treatment is completed and the desired microstructure obtained, a circular notch is machined on each specimen, and the samples are subjected to push-pull fatigue loading. Several combinations of notch and microstructural sizes have been tested. This method provides an easy way to record and analyse the effect of the microstructure upon crack growth rate. It was observed that the space between successive crack-tip arrests correlates well with the material grain size. Another interesting observation is that in the majority of the cases studied the cracks did not initiate at the point of maximum stress concentration. This is surprising since the classical methods of notched fatigue limit analysis clearly indicate the horizontal symmetry axis as the initiation and propagation direction for pushpull loading.
\end{abstract}

KEYWORDS. Short crack; Microstructural barrier; Grain size; Notch; Fatigue crack initiation; Fatigue limit; Crack growth rate.

\section{INTRODUCTION}

ᄀ he topic of this conference is the characterization of the crack tip stress and strain fields. The inauspicious beginnings of the characterization of fatigue crack growth rate of long cracks by the stress intensity factor range as described in the Paris law is a well-known episode in fracture mechanics lore. There, a single parameter embodies the physics of the problem and crack growth rate can be expressed as a unique function of $\Delta \mathrm{K}_{\mathrm{I}}$ irrespective of the geometry of the component or the type of load applied. But Paris law breaks down for small cracks.

Microstructurally short fatigue crack growth is a prime example of a situation where a single parameter characterization of the crack stress and strain fields does not seem to be entirely appropriate. It is a very important problem from the practical point of view, for there are many situations where the life of the component is "decided" when the crack length is of the order of the grain diameter. This is particularly the case in long-cycle fatigue problems when loading close to the fatigue limit. It is now well established that the fatigue limit in metallic materials is really a threshold condition for the propagation of very small cracks. Experimental evidence has shown that, in plain specimens subject to uniaxial loads, cracks form on persistent slip bands (PSBs) and grow along them. At stresses below the fatigue limit, cracks start growing fast but then they stop and become non-propagating. At stresses just above the fatigue limit, cracks decelerate and may temporarily halt a number of times, but they do not stop growing altogether. Later on they accelerate and finally reach a regime of apparent continuous propagation. Microscopic observations have identified the locations of minimum crack growth as microstructural barriers to slip propagation such as grain or phase boundaries. The crack decelerates on 
approaching the grain boundary until a new slip band is initiated in the neighbouring grain, along which the crack will propagate next. This nucleation process of slip bands has to be repeated afresh in each grain.

The study of the fatigue limit of notched components is another case of interest. Here the problem is one of short crack growth, with the additional difficulty that the stress field through which the crack is growing has usually a very steep gradient. It has been found that in sharp notches it may be possible for a crack to grow through a few grains and then to become non-propagating. This suggests that the critical event in notch fatigue may not be the initiation of the crack itself, but rather the relative capacity of this crack to overcome successive microstructural barriers when the stresses that are driving it diminish rapidly. Typical non-propagating cracks found in sharp notched specimens of carbon steel have lengths of the order of a few tens of microns.

We have devised [1] a simple experimental technique whereby all this can be studied with an unprecedented level of ease and detail. The innovative aspect of this technique is the use of specially developed test coupons with grain sizes of a few millimeters - or even centimeters - and the use of low magnification USB cameras by means of which the crack growth process and the interactions with the microstructure can easily be registered and examined. Digital image correlation techniques have also been employed to enhance the technique.

\section{EXPERIMENTAL PROCEDURE}

\section{Thermo-mechanical treatment}

tudies carried out by the research group of the University of Seville [2], have shown that it is possible to produce a substantial increase in the grain size of commercially pure aluminium sheets by means of a combination of two thermal treatments and an intermediate moderate cold working. The process is simple enough and easy to control, and it provides highly repeatable results. Aluminium 1050 Puraltok 99.5-H24 by Alu-Stock is used in sheets of $4.0 \mathrm{~mm}$ thick. Chemical composition: 99.56; Cu: 0.08; Fe: 0.2; Si: 0.1. The aluminum sheets are cut into pieces of 45x300 mm, in parallel to the lamination direction. A tubular furnace is used for the thermal treatments (Carbolite model 215GHA12).

The aim of the first thermal treatment is to obtain a deformation-free equiaxial structure; different treatments were tested by changing the recrystallization temperature, the heating rate and the interval at constant temperature. After observing the resulting microstructures, a heating rate of $2.6^{\circ} \mathrm{C} / \mathrm{min}$ was chosen, from ambient temperature to $550^{\circ} \mathrm{C}$. The constant temperature has to be kept during 5 hours, followed by air cooling. After 5 hours, the surface grains become larger compared to the inner grains, and the larger size along the specimen creates a non uniform deformation along the specimen thickness at the following stage (mechanical treatment). Next, cold working is performed in a MTS 810 . The deformation level applied at this stage will determine the size of the grain after second recrystallization.

The following deformation percentages were chosen: $0-8-11-14-18 \%$. This treatment is carried out by a MTS 810 controlling each deformation. $0 \%$ corresponds to the material undergoing only the first recrystallization. The third stage is again a temperature ramp from $\mathrm{T}_{\text {amb }}$ to $\mathrm{T}=550{ }^{\circ} \mathrm{C}$ at a rate of $2.6^{\circ} \mathrm{C} / \mathrm{min}$; then, the temperature is to be kept constant during 15 hours and that it is increased again to $575^{\circ} \mathrm{C}$ and kept at that temperature during 1 hour. Finally, it is air cooled. At this stage new crystals growing at the expense of the old ones are formed. The final size obtained depends largely on the level of plastic deformation applied. Figure 1 shows an example of the possible microstructures obtained and the corresponding level of total deformation applied, the upper image corresponds to a specimen which has undergone $8 \%$ of total strain during the mechanical treatment and the bottom image corresponds to a specimen subjected to $14 \%$ of total strain.

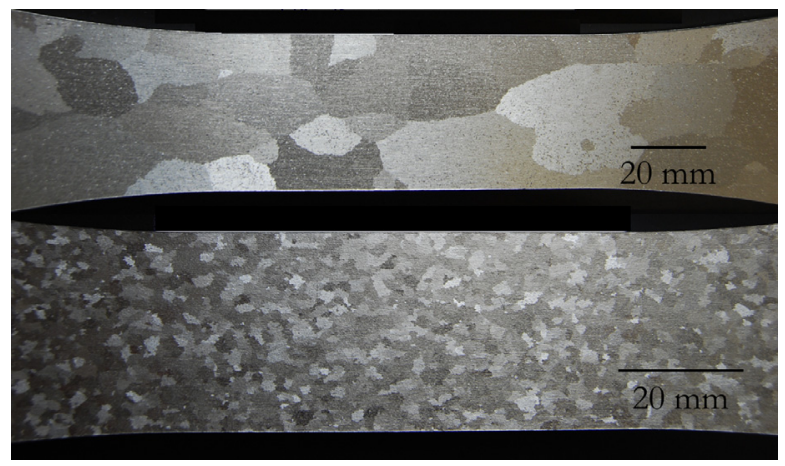

Figure 1: Microstructure obtained depending on the degree of applied strain. 


\section{Grain size}

The grain size measurement is made according to ASTM E112, using software Simagis Live and following the procedure described in [16]. The obtained grain size values are summarized in table 1

\begin{tabular}{cc}
\hline Deformation [\%] & Grain Size $[\mathrm{mm}]$ \\
0 & 0.066 \\
18 & 0.394 \\
14 & 1.41 \\
11 & 3.46 \\
8 & 9.74 \\
\hline
\end{tabular}

Table 1: Grain size obtained in each thermo-mechanical treatment

\section{Testing machine}

Once the thermo-mechanical treatment is completed and the desired microstructure obtained, a circular notch is machined on each specimen, and the samples are subjected to push-pull fatigue loading. Several combinations of notch and microstructural sizes have been tested.

A RUMUL resonant testing machine (Testronic $100 \mathrm{kN}$ ) was used for fatigue testing. All tests were load-controlled, with $\mathrm{R}=0.1$ (pull-pull) and maximum stress between 45 and $95 \mathrm{MPa}$. Resonance frequencies for the loading conditions and geometry of specimens are between 75 and $90 \mathrm{~Hz}$. Crack propagation results in a decrease on the resonance frequency. After several trials, it was established that a frequency decrease of $0.7 \mathrm{~Hz}$ was a good indicator of impending failure. A larger decrease results in complete failure, where the crack faces rapidly separate and the remaining section is plastically deformed, making post mortem analysis impossible.

\section{Crack Follow-up}

An experimental procedure for measuring crack length and growth was developed in order to observe crack propagation and interactions with the microstructure. Two low magnification optical microscopes showing real time video images were connected to a computer via USB. They are easily mounted onto a platform and, as shown in figure 2, they are set to cover the surface of both sides of the specimen simultaneously. The magnification is adjusted by regulating the distance between the magnifier and the specimen.

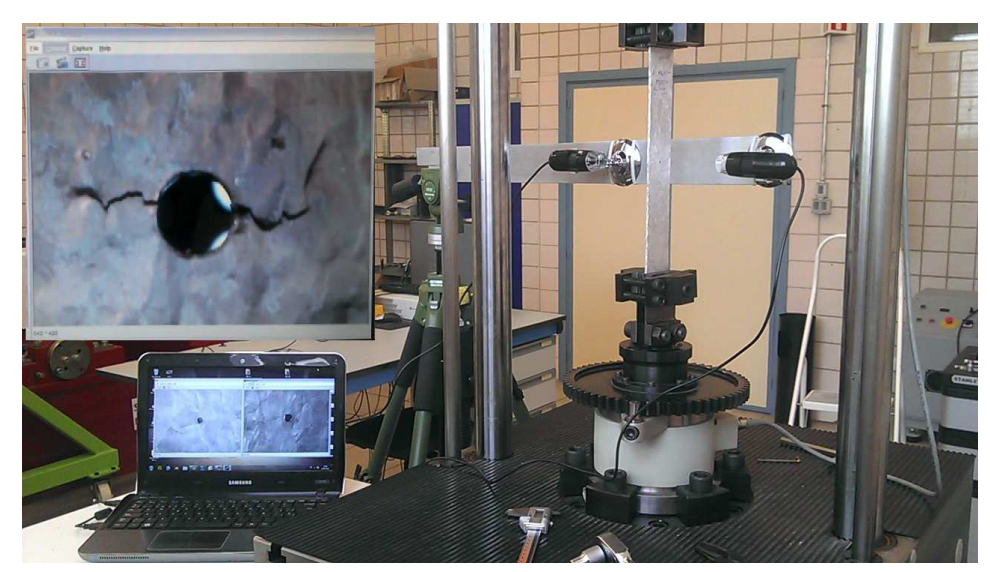

Figure 2: Crack Follow up Set up.

Since the materials used have low mechanical properties and strains are always kept in elastic region, the movements produced during specimen load and unload are very small; as a result, the recorded video is quasi-static and there is no need to stop the test, measure the crack length and resume. This facilitates the crack growth analysis, and largely reduces the amount of time needed for the test in contrast with other crack follow up experimental techniques, such as replica with acetate. This technique makes it possible to keep a record of every test and of any possible potential crack on both 
sides of the specimen. This is important because studies can be made either of the interaction between the crack and the microstructure and of the interaction between different cracks and different crack branches. A frame of the video is taken every 30 seconds in order to measure crack length. Next, the crack length is measured through the image analysis free software ImageJ.

The combination of this information with the test frequency is elaborated in graphics of crack lengths depending on the number of cycles. On the basis of this graphic, the graphic on growth rate depending on the number of cycles and/or the crack length is elaborated.

It is certain that the growth rate is generally expressed in relation to the parameter $\mathrm{K}$ or the crack length; however, if it is expressed in relation to the number of cycles, the growth rates of the different cracks can be compared at the same point in time.

\section{RESULTS}

$\mathrm{F}$ ig. 3 shows a photograph extracted from one of the fatigue tests videos. The crack grows from a circular notch with a radius of $1 \mathrm{~mm}$. The grain size is of $9.74 \mathrm{~mm}$. The test is load controlled, type stress-stress $(\mathrm{R}=0.1)$ with a maximum stress of $45 \mathrm{MPa}$; under these loading conditions and geometry of specimen, the resonance frequency is $82.13 \mathrm{~Hz}$. Fatigue life is $4.09 \times 10^{6}$ cycles. The left image corresponds to one of the sides of a sample and the right image corresponds to the other face. The latter is shown from an inverted angle, thus, both left cracks correspond to the same side of the notch, and similarly, both cracks growing from the right side correspond to the other side of the notch. In the video, it is possible to observe crack tip arrest at grain boundaries, as well as changes in growth direction.

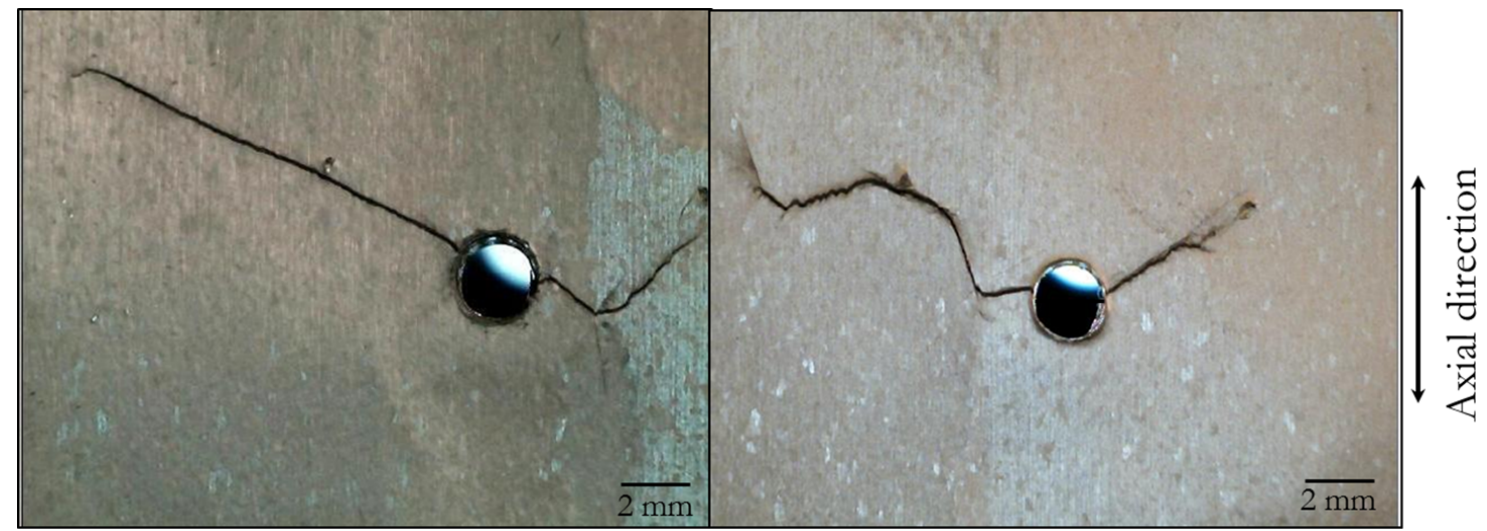

Figure 3: Crack growing from a circular notch with a diameter of $2 \mathrm{~mm}$ within a microstructure of $9.74 \mathrm{~mm}$.

As mentioned above, an extract of video is taken at a specific time interval (e.g. 30 seconds) in order to measure crack length and growth rate. The number of cycles is calculated on the basis of the test frequency. The crack length is measured through the use of ImageJ. Figure 4 shows crack length in relation to the number of cycles for three cracks growing from the same notch. All of them show acceleration and deceleration patterns along their trajectory.

Figure 5 shows growth rate in relation to crack length for three cracks growing in different microstructures with grain sizes of $1.41,3.46$ and $9.74 \mathrm{~mm}$. There is a clear correlation between the average spacing of the consecutive crack halts and the grain size of the material.

An interesting observation is that in the majority of the cases studied the cracks did not initiate at the point of maximum stress concentration. Recall that we are referring to push-pull loading here. This is surprising since the classical methods of notched fatigue limit analysis clearly indicate the horizontal symmetry axis as the initiation and propagation direction.

Figure 6 shows the results of measurements of the location of the crack initiation point around the circumference of the notch for different holes radii and for several specimens with the same microstructural size of $9.74 \mathrm{~mm}$. On the left, a diagram shows a polar coordinate system corresponding to a quarter of the notch and it presents the crack initiation points. The graphic on the right shows the corresponding frequency distributions. Approximately 36 measurements were made for each notch radius.

The following trend appears. When the size of the notch diminishes (in relation to the microstructure) the width of the frequency distribution seems to become larger and the most frequent location of the initiation point value moves away 
from the horizontal axis. Compare the values of 10, 15 and approximately 30 degrees obtained for notches of radii 2, 1 and $0.5 \mathrm{~mm}$ respectively.

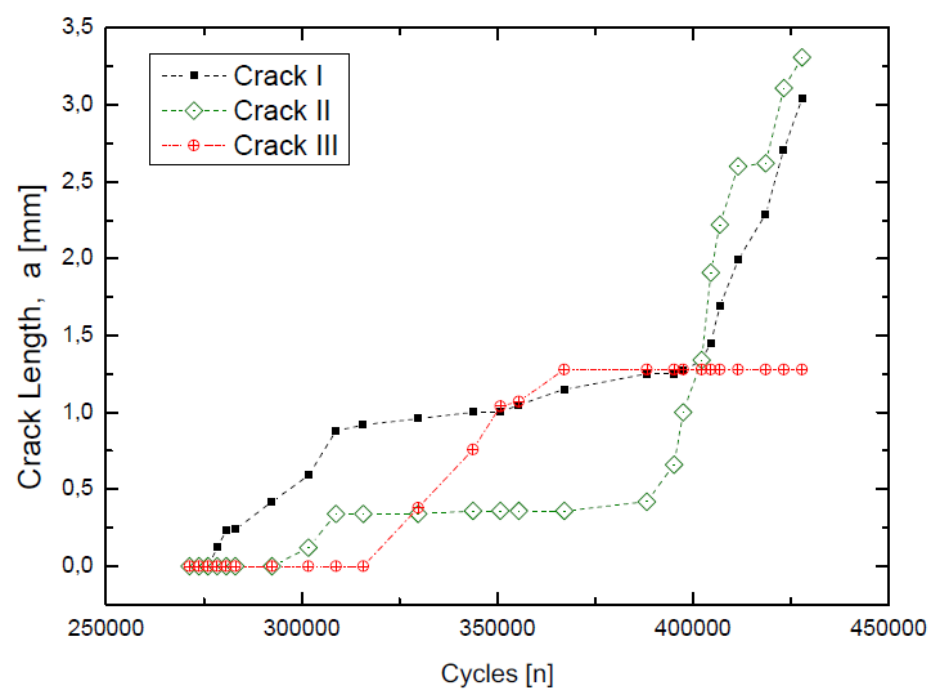

Figure 4: Crack length in relation to number of cycles.

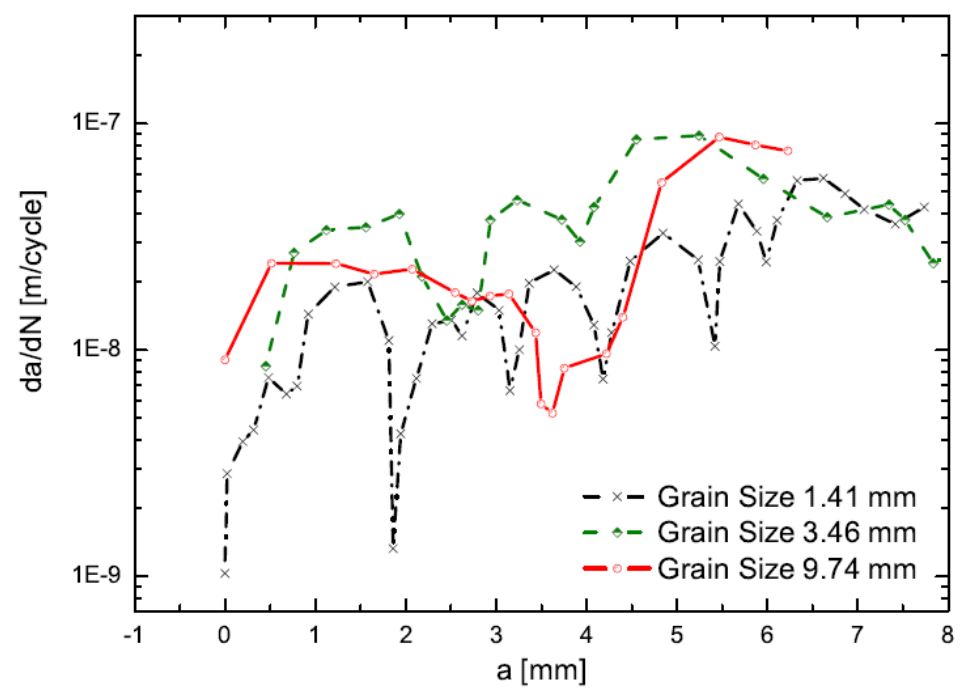

Figure 5: Crack growth in relation to crack length.

Conversely, bigger notch radii tend to concentrate the possible initiation area bringing it closer to the horizontal axis. Of course, for materials with "normal" microstructural sizes of the order of a few tens of microns, and "normal" notch sizes of a few millimeters, where the notch is several times larger than the microstructure, this indicates that cracks would normally initiate in the expected horizontal symmetry axis location, which is just as well.

This observation clearly reveals that the application of the usual techniques of notch analysis (viz critical distance [3,4] or notch sensitivity index concepts $[5,6]$ ) may not be applicable in situation where the notch size is of the same order or even smaller that the microstructure and this points out that this region of notch size may be a good testing ground to discriminate among different notch fatigue calculation methods.

Finally, we would just like to present some preliminary results obtained by using Digital Image Correlation techniques in our experiments with the big grains material.

Figure 7 shows the resulting deformation areas for a) Specimen with a microstructure of $0.06 \mathrm{~mm}, 2 \mathrm{~mm}$ notch size and subjected to a $50 \mathrm{MPa}$ tension-tension ( $\mathrm{R}=0.1)$ fatigue loading, with $\mathrm{N}=7.9 \times 10^{5}$ cycles and b) microstructure of $8.22 \mathrm{~mm}$, $2 \mathrm{~mm}$ notch size and subjected to a $45 \mathrm{MPa}$ tension-tension $(\mathrm{R}=0.1)$ fatigue loading, with $\mathrm{N}=2.38 \times 10^{6}$ cycles. For carrying out the DIC analysis the fatigue test is interrupt every 10000 cycles and a photograph of the speckle pattern is taken. Later these images are analyzed with the VIC 2D software in order to obtain the evolution of the strain throughout the test. 

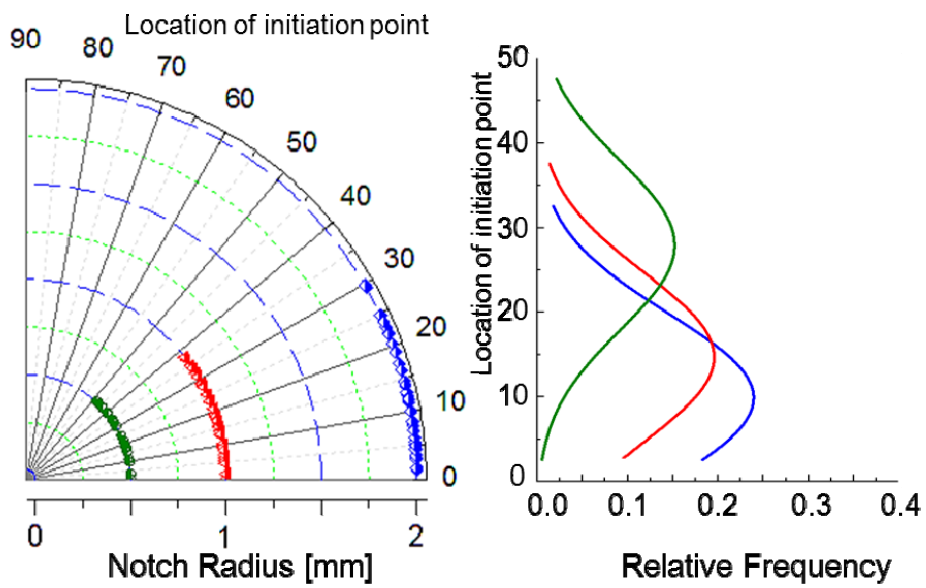

Figure 6: Location of crack initiation point in relation to notch size for a microstructure of $9.74 \mathrm{~mm}$.

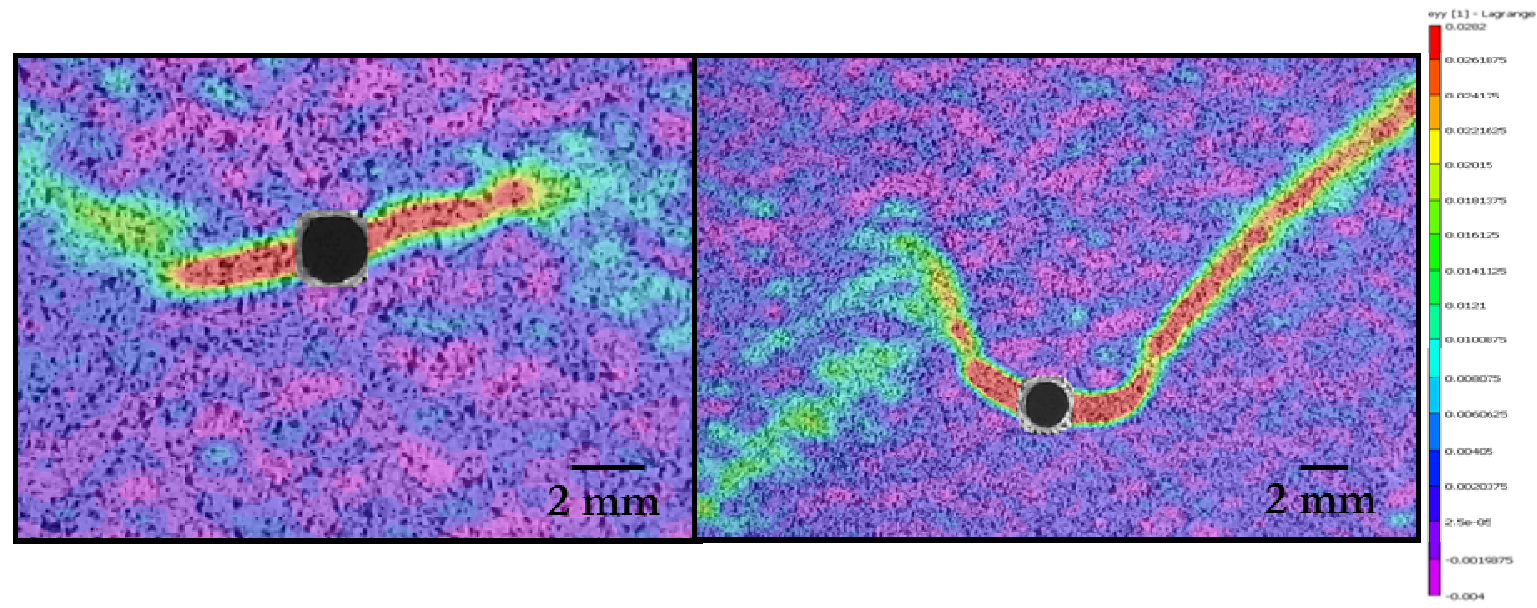

Figure 7: Deformation area as a result of crack growth for cracks growing in microstructures of 0.07 and $8.22 \mathrm{~mm}$.

Both pictures show the strain state (calculated on the loading direction, $\varepsilon_{y y}$ ) of the sample at the moment of the initiation of plastic collapse. It is possible to observe the effect of the grain size on the deformation area, as a result of crack growth. In the case of tests conducted with small microstructures, symmetrical deformation bands are observed on both sides of the notch. While increasing the grain size, the deformation bands are influenced by the microstructure.

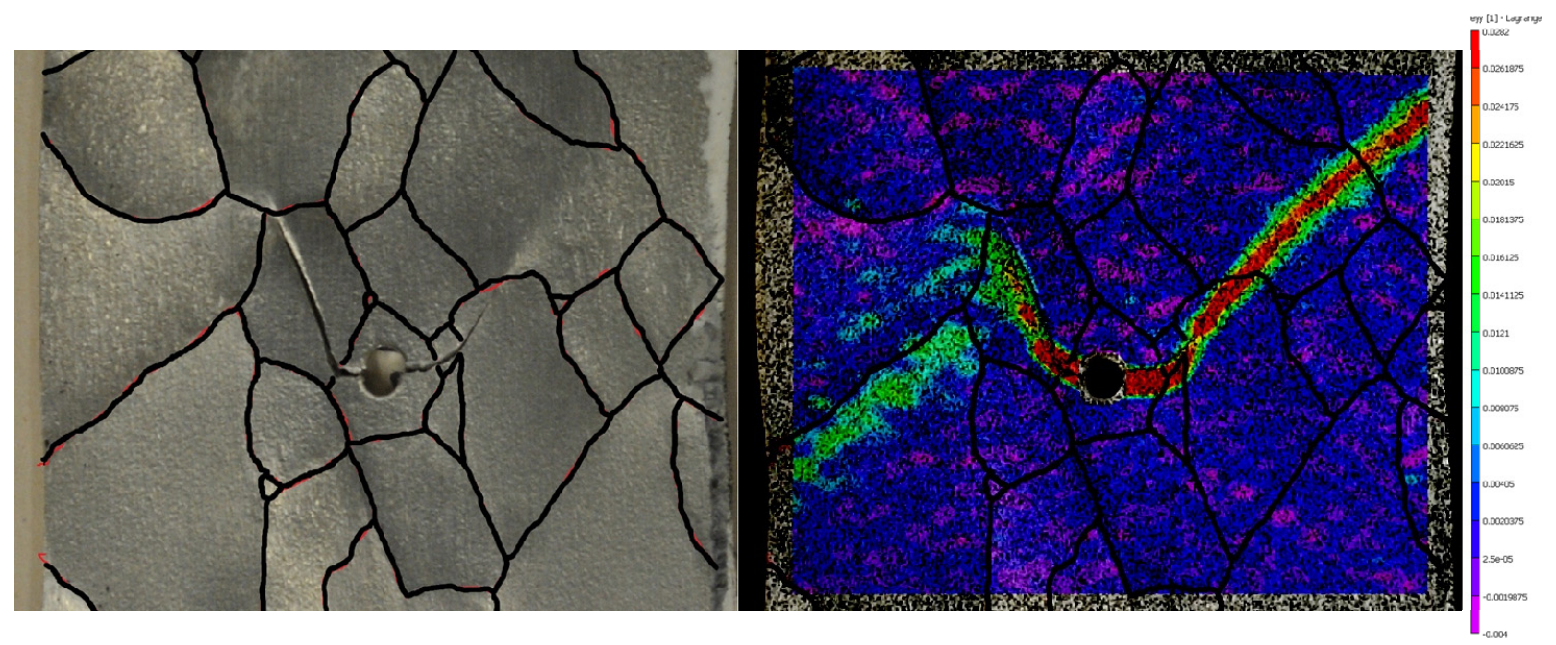

Figure 8: This image shows the DIC analysis and microstructure superposition. 
In the case of large microstructures, the grains traverse the entire specimen thickness $(2 \mathrm{~mm}$.). This fact allows studying the crack propagation through the DIC analysis on one side of the specimen and by the image analysis method explained above on the other side of the specimen. Figure 8 shows a comparison between the microstructure analysis and the Digital Image Correlation analysis. It is possible to observe how the left crack and its plastic zone is arrested at a grain boundary and a new plastic zone is generated not in the contiguous grain but in another neighboring grain that it is probably better oriented according to the loading direction.

We are currently working on refining the speckled pattern in order to measure with more accuracy the spreading and width of the deformation bands.

\section{CONCLUSIONS}

7 he experimental set-up needed for crack follow-up is very simple and the tests can be developed easily. As a result, a large number of samples and test conditions can be analyzed in a relatively short period of time.

As the studies have shown, the behaviour of the very long "short" cracks observed in the materials with the very big grains procedure is similar to that observed when "normal", smaller microstructures are considered $[7,8]$.

As the grain size increases, cracks presenting sizes commonly associated with specific behaviours according to MFLE, begin to behave largely in accordance with the principles of microstructural fracture mechanics.

Also, it was observed that for small radii of the notch and large grain sizes there is a wide region along the notch surface where the likelihood of initiation is equally high. The smaller the notch radius, the wider the possible region of initiation and the more it deviates from the horizontal symmetry plane.

This experimental technique may be useful for understanding the behaviour of short cracks growing from small stress concentrators (inclusions, bubbles, pits, etc.) existing in materials with smaller grain sizes but presenting similar notchgrain size relation.

\section{ACKNOWLEDGEMENTS}

he authors wish to thank the Ministry of Education and Sciences of Spain for providing the financial support through project DPI2008-01100 and DPI2011-27019.

\section{REFERENCES}

[1] Lorenzino, P., Navarro, A., Krupp, U., Naked eye observations of microstructurally short fatigue cracks. Submitted for publication to Int. J. of Fatigue (2013).

[2] Lorenzino, P., Fatiga en componentes con concentradores de tención bajo carga en modo I, Ph.D. Thesis, University of Seville, Spain, (2012).

[3] Taylor, D., Geometrical effects in fatigue: a unifying theoretical approach, Int. J. of Fatigue, 21 (1999) 413-20.

[4] Taylor, D., The theory of critical distances: a new perspective in fracture mechanics, Elsevier, (2007).

[5] Peterson, RE, Notch sensitivity. In: Sines G, Waisman JL, Eds. Metal Fatigue, McGraw-Hill, (1959) 293-307.

[6] Neuber, H., Kerbspannungslehre. Springer Verlag; (1937). Translated into English, Theory of Notches, Edwards, J. W., Ann Arbor, MI, (1946).

[7] Herbig, M., King, A., Reischig, P., Proudhon, H., Lauridsen, E. M., Marrow, J., Buffière, J-Y, 3-D growth of a short fatigue crack within a polycrystalline microstructure studied using combined diffraction and phase-contrast X-ray tomography, Acta Materialia, 59 (2011) 590-601.

[8] Krupp, U., Düber, O., Christ, H.-J., Künkler, B., Köster, P., Fritzen, C.-P., Propagation mechanisms of microstructurally short cracks factors governing the transition from short- to long- crack behavior, Materials Science and Engineering A, 462 (2007) 174-177. 